LA-UR- $98-2126$ Title:

Author(s):

Submitted to:

\section{Los Alamos} NATIONAL LABORATORY
SUBSTORM EFFECTS IN MHD AND TEST PARTICLE
SIMULATIONS OF MAGNETOTAIL DYNAMICS

$$
\text { CONF-9805129-- }
$$

Joachim Birn, NIS-1

Michael Hesse, NASA Goddard Space Flight Center

Proceedings of ICS-4

Terra Scientific Publishing Company

Tokyo, Japan

May 15, 1998

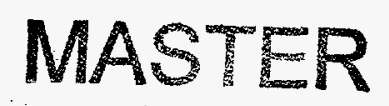

DISTABUTION OF THS DOOUMENT IS UNHWHTEO

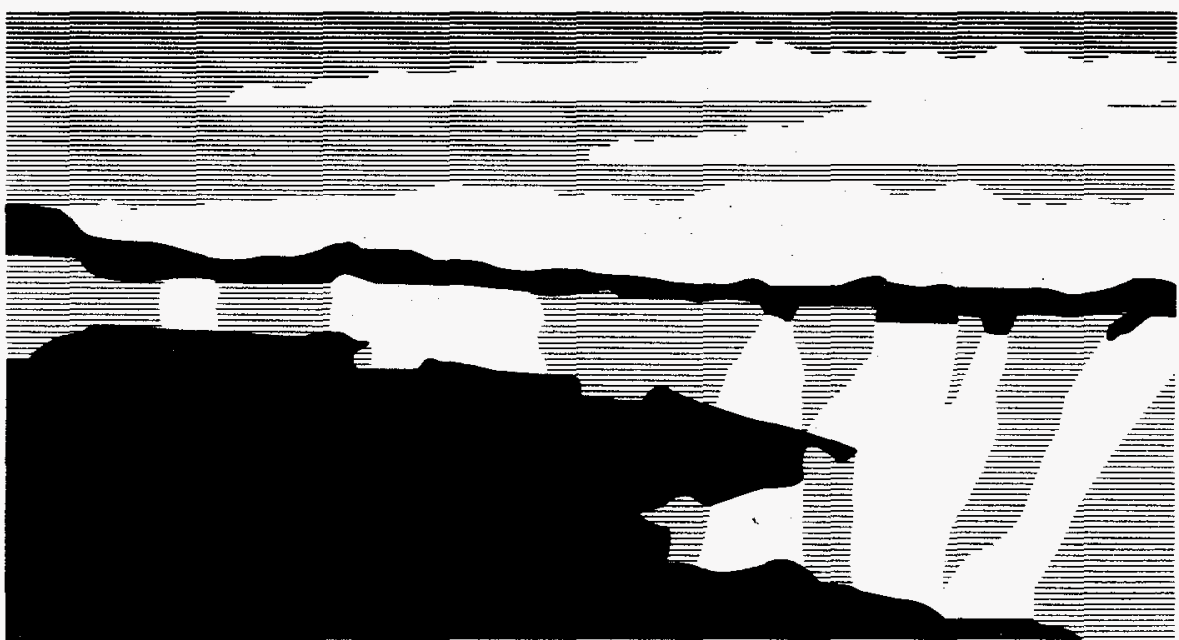

Los Alamos National Laboratory, an aftirmative action/equal opportunity emplóyer, is operated by the University of California for the U.S. Department of Energy under contract W-7405-ENG-36. By acceptance of this article, the publisher recognizes that the U.S. Government retains a nonexclusive, royalty-free license to publish or reproduce the published form of this contribution, or to allow others to do so, for U.S. Government purposes. The Los Alamos National Laboratory requests that the publisher identify this article as work performed under the auspices of the U.S. Department of Energy. 


\section{DISCLAIMER}

This report was prepared as an account of work sponsored by an agency of the United States Government. Neither the United States Government nor any agency thereof, nor any of their employees, makes any warranty, express or implied, or assumes any legal liability or responsibility for the accuracy, completeness, or usefulness of any information, apparatus, product, or process disclosed, or represents that its use would not infringe privately owned rights. Reference herein to any specific commercial product, process, or service by trade name, trademark, manufacturer, or otherwise does not necessarily constitute or imply its endorsement, recommendation, or favoring by the United States Government or any agency thereof. The views and opinions of authors expressed herein do not necessarily state or reflect those of the United States Governmeat or any agency thereof. 


\section{DISCLAIMER}

Portions of this document may be illegible in electronic image products. Images are produced from the best available original document. 


\title{
SUBSTORM EFFECTS IN MHD AND TEST PARTICLE SIMULATIONS OF MAGNETOTAIL DYNAMICS
}

\author{
J. Birn \\ Los Alamos National Laboratory, Los Alamos, New Mexico \\ M. Hesse \\ NASA Goddard Space Flight Center, Greenbelt, Maryland
}

\begin{abstract}
Recent magnetohydrodynamic simulations demonstrate that a global tail instability, initiated by localized breakdown of MHD, can cause plasmoid formation and ejection as well as dipolarization and the current diversion of the substorm current wedge. The connection between the reconnection process and the current wedge signatures is provided by earthward flow from the reconnection site. Its braking and diversion in the inner magnetosphere causes dipolarization and the magnetic field distortions of the current wedge. We demonstrate the characteristic properties of this process and the current systems involved. The strong localized electric field associated with the flow burst and the dipolarization is also the cause of particle acceleration and energetic particle injections. Test particle simulations of orbits in the MHD fields yield results that are quite consistent with observed injection signatures.
\end{abstract}

\section{Introduction}

Magnetospheric substorms are most commonly interpreted in terms of two paradigms, depicting changes in the inner tail and the more distant tail, respectively. The "substorm current wedge" picture [e.g., McPherron et al., 1973] suggests that a dynamic process in the inner tail leads to a reduction or disruption [e.g., Lui et al., 1990] of the cross-tail current and its diversion, via field-aligned currents, to the ionosphere, where the currents close through the auroral electrojet. The changes in the more distant tail are most frequently interpreted in terms of plasmoid formation and ejection [e.g., Hones, 1977], caused by reconnection in the inner tail. While both pictures are widely accepted, the connection between the two paradigms and the initiation process for the dynamic evolution are still a matter of debate. In this paper we will discuss recent magnetohydrodynamic (MHD) simulations that demonstrate how the two pictures might be connected within one large-scale instability, triggered by the localized breakdown of ideal MHD. This simulation also includes the generation of a localized electric field, associated with the collapse and dipolarization of the inner tail, which is reponsible for charged particle acceleration. Tracing test particles in these MHD fields has lead to an improved understanding and modeling of particle energization and injections of energetic particles in the inner magnetosphere [Birn et al., 1997b, 1998a; see also Reeves, 1998].

In the following section we will present basic properties of the simulation which are important to understand the time sequence and the connection between macro- scopic effects. Section 3 will focus on new details of the current diversion associated with the substorm current wedge [Birn et al., 1998b], which demonstrate that additional current systems are necessary for a full understanding of its properties. Section 4 is devoted to energetic particle properties, obtained from tracing test particles in the fields of the MHD simulation. These simulations reproduce characteristic properties of energetic particle injections as observed at geosynchronous orbit [e.g., Bim et al., 1997a].

\section{MHD Simulation, Overview}

The three-dimensional MHD simulation that models the dynamic evolution of the magnetotail [Birn and Hesse, 1996] starts out from an initial state that includes the transition region between the Earth's dipole and the magnetotail in a modeling region from $x=-5 R_{E}$ tailward. As there are no self-consistent equilibrium models valid for this region, we started from the superposition of a dipole field and a self-consistent tail equilibrium [Birn, 1987] and used a relaxation technique to generate an equilibrium.

Modeling effects of the substorm growth phase, we subsequently applied an external electric field (or rather the corresponding plasma inflow) at the high-latitude boundary of the simulation box to cause the formation of a thin current sheet in the inner tail. Such thin current sheets are observed in this region late in the substorm growth phase [e.g., Sanny et al., 1994]; they are presumably crucial in the initiation of the substorm expansion phase [e.g., McPherron et al., 1987; Schindler and Birn, 1994].

The expansion phase is initiated by imposing finite resistivity, while the external electric field is turned off. Anomalous resistivity is chosen to model the consequences of a yet unknown collisionless dissipation mechanism breaking the ideal MHD constraint. As a result the configuration becomes unstable to a generalized three-dimensional tearing mode, which leads to the formation and tailward ejection of a plasmoid. The time $t=0$ corresponds to the onset of resistivity; $t<0$ represents the period of formation of the thin current sheet. The dynamic evolution for $t>0$ is characterized by the formation and tailward motion of a plasmoid with a further intensification of the current density in the vicinity of the reconnection site (near-Earth neutral line at $x \approx-23 R_{E}$ ). The neutral line forms at $t \approx 3 \mathrm{~min}$, the cross-tail electric field, the earthward flow, and the (region 1 type) field-aligned current start to rise at $t \approx 5-6 \mathrm{~min}$ and maximize by $t=8$ $\min$. 

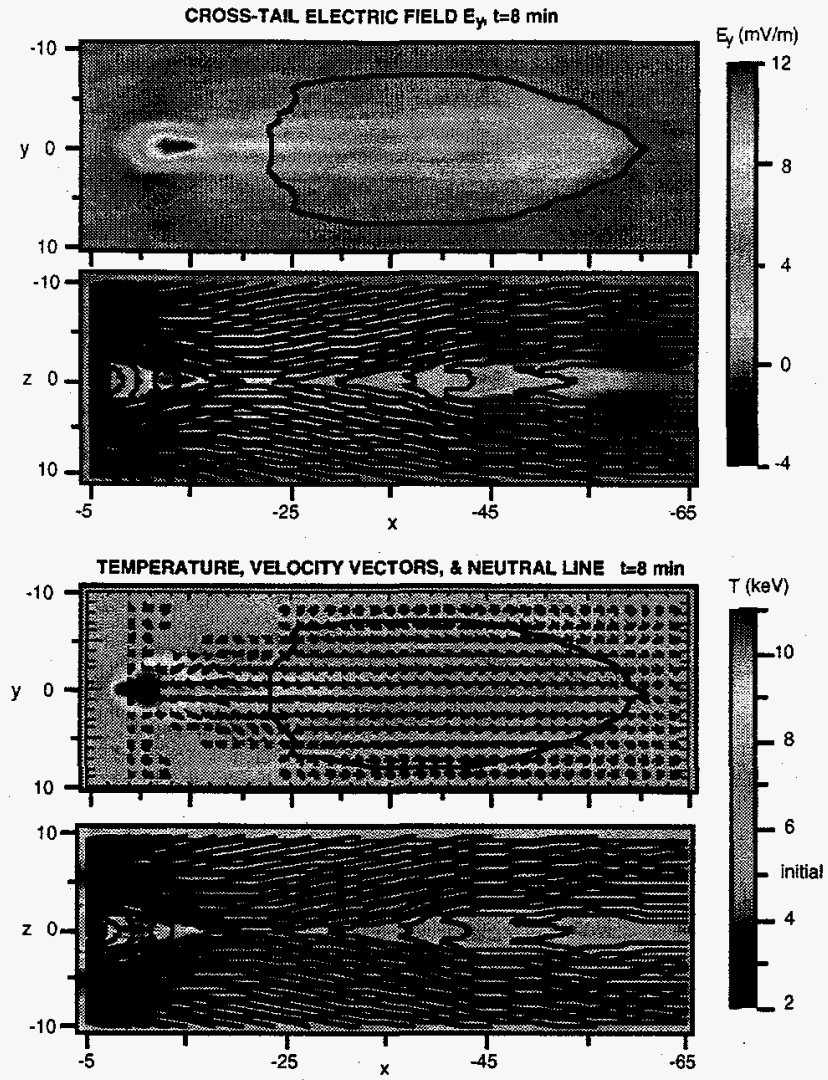

Figure 1. Magnitude of the cross-tail electric field (colorcoded) at $t=8 \mathrm{~min}$ in the equatorial plane (top) and the midnight meridian plane (bottom) obtained from the MHD simulation [Birn and Hesse, 1996], used as the basis for the test particle study. Also shown are the magnetic neutral line in the $x, y$ plane and magnetic field lines in the $x, z$ plane.

Figure 1 demonstrates characteristic increases of the cross-tail electric field and the plasma temperature, both in the midnight meridional plane and in the equatorial plane, at $t=8 \mathrm{~min}$. It is remarkable that the predominant effects are significantly earthward from the neutral line. They occur in the region of strong dipolarization, where the earthward flow (superposed on the color-coded temperature in panel 3) is braked and diverted. The temperature increase in the inner tail by a factor of about 2 is quite consistent with observations [Baumjohann et al., 1991; Huang et al., 1992; Birn et al., 1997a]. It is due to a combination of resistive, nonadiabatic heating and adiabatic compression.

\section{Current Diversion}

Current diversion in simulations of magnetotail dynamics has been discussed previously by Birn and Hesse [1991, 1996], Hesse and Birn [1991], and Scholer and Otto [1991]. Figure 2 provides an overview of the regions of strong current diversion and their association with the braking and diversion of earthward flow caused by reconnection, taken from a recent discussion by Birn et al. [1998b]. Color-coding shows the divergence of the parallel current, integrated over $z$, as defined by

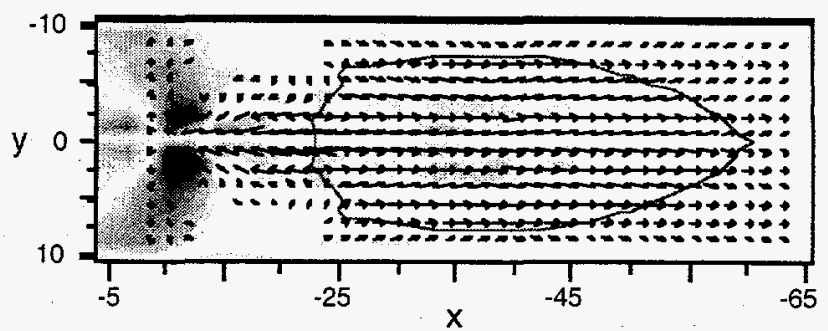

Figure 2. Divergence of parallel current (color-coded), flow vectors, and magnetic neutral line (red line) in the equatorial plane at $t=8 \mathrm{~min}$. The current densities are integrated over $z$ from $z=0$ to $z=z_{\max }$ to include the dominant contributions outside the equatorial plane. Only flow vectors with a minimum magnitude of $0.08(\sim 80 \mathrm{~km} / \mathrm{s})$ are shown. The figure illustrates the relationship between earthward flow, generated at an $\mathrm{x}$ line near $x=-23$, its braking and diversion between $x$ $=-10$ and $x=-18$, and the generation of region 1 type fieldaligned currents.

$$
\nabla \cdot \mathbf{J}_{\|}=\int_{0}^{z_{\max }} \nabla \cdot \mathbf{j}_{\|} d z
$$

where $\mathbf{j}$ denotes the current density. As in panel 3 of Figure 1 , we overlaid the velocity vectors and the magnetic neutral line (solid red line) onto the color-coded values of $\nabla: \mathbf{J}_{\|}$. Here red corresponds to diversion to earthward fieldaligned currents, while blue corresponds to diversion to tailward currents. The main region of current diversion between $x \approx-10$ and $x \approx-13$ therefore represents the generation of the region 1 system of the substorm current wedge, colocated with the region of flow braking and diversion. The sense of the field-aligned currents is consistent with the one expected from the shear or twist of the magnetic field lines caused by the flow vorticity in this region. It is interesting that there are regions of opposite (that is, region 2 sense) field-aligned current generation closer to the Earth. This can be associated with an opposite vorticity at the earthward edge of the diverted flow.

The changes in the currents cannot be represented by just one current system, the traditional current wedge, alone. Additional current perturbations found in our simulation are demonstrated in Figure 3, which shows the changes in the current density vectors between $t=0$ and $t=9 \mathrm{~min}$ for two different cross-sections, (top) $x=-8.75$ and (bottom) $y=-2$. At $x=-8.75$ perturbed currents flow from dusk to dawn close to the equatorial plane, reducing the preexisting cross-tail current as in the current wedge, combined with duskward currents at higher latitude. This system simply results from the expansion of the plasma/current sheet in the dipolarizing region. At $y=-2$ we see that the earthward flowing field-aligned currents of the current wedge are also fed in part by radial currents from the vicinity of the equatorial plane. One system comes from closer to the Earth; it is possibly connected with the outward flowing (that is, region 2 type) field-aligned current system in the inner tail, indicated in Figure 2. Another contribution stems from farther out, closing at higher latitude, again with an outward flowing field-aligned current. This system remains here confined in the tail region and does not necessarily connect with the ionosphere. The two systems that close in the tail may be relevant for Mercury's magnetosphere, where wedge currents cannot close through ionospheric currents as in the Earth's case. 

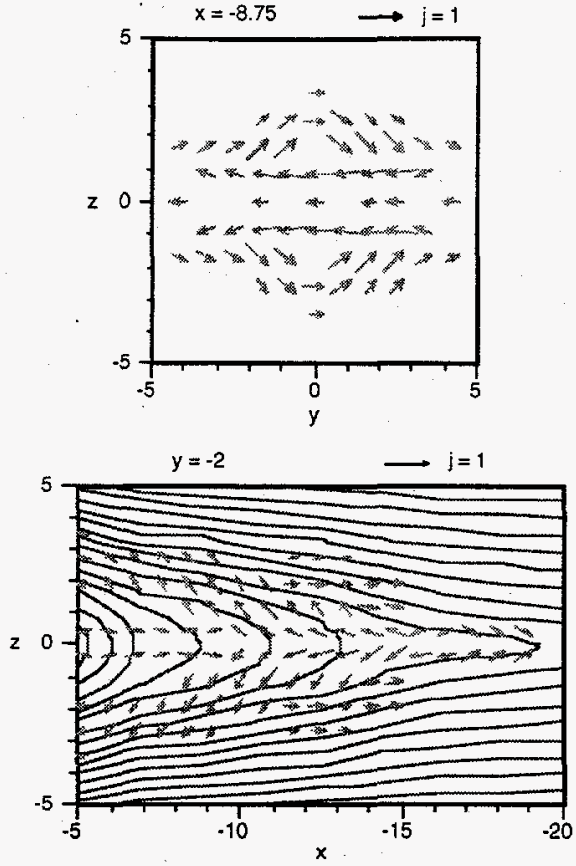

Figure 3. Current perturbations associated with the substorm current wedge. The figure shows the difference between the current density vectors at $t=9 \mathrm{~min}$ and $t=0$ for two cross sections of the simulation box at (top) $x=-8.75$ and (bottom) $y=-2$. The unit length vectors correspond to a current density of $10^{-8} \mathrm{~A} / \mathrm{m}^{2}$. For clarity, only vectors with a minimum length of (top) 0.2 and (bottom) 0.1 are shown. The bottom panel also shows the magnetic field direction in the plane $y=-2$ (thin lines).

Inertia-driven currents [Haerendel, 1992; Shiokawa et al., 1997] are only minor contributions to the diversion into the total field-aligned currents of the current wedge, despite the crucial role of the flow braking and flow diversion in distorting the magnetic field and thereby increasing the field-aligned currents. The dominant role is played by the pressure gradient driven contributions, which change consistently with the distorted magnetic field. Here the terms that involve $z$ gradients dominate [Birn et al., 1998b], rather than azimuthal [Shiokawa et al., 1998] or radial pressure gradients.

Figure 4 shows the contributions to the diversion of perpendicular currents as a function of $x$. These contributions are now also integrated over $y$ (here for the dawn sector). The term $\nabla \cdot \mathbf{I}_{1}$ represents the total divergence of the parallel currents, $\nabla \cdot \mathbf{I}_{\mathrm{x}}$ and $\nabla \cdot \mathbf{I}_{\mathrm{y}}$. represent the divergence of radial $(x)$ and cross-tail $(y)$ perpendicular currents, respectively, and the subscripts $p$ and $v$ refer to pressure gradient and inertia contributions. The $x$ component of the divergence of the inertia current and all $z$ components are very small and therefore not shown. Diversion to fieldaligned currents starts inward of $x=-15$ but ceases earthward of $x=-7.5$. Just tailward of $x=-15$ there is a contribution from the inertia currents. This contribution, however, is essentially canceled by an oppositely directed contribution from radial currents driven by pressure gradients. Just inward of $x=-15$ the diversion of the cross-tail component dominates, whereas farther earthward the diversion of the radial component becomes more important.

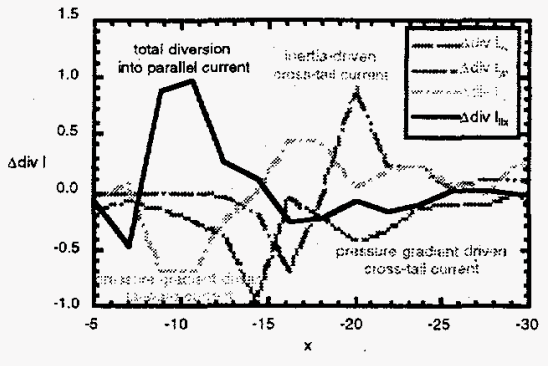

Figure 4. Dominant contributions to the changes of the diversion to field-aligned currents between $t=9 \min$ and $t=$ 0 . $I_{y v}$ corresponds to the cross-tail inertia current, $I_{x p}$ and $I_{y p}$ correspond to the pressure gradient currents in the $x$ and $y$ direction, and $I_{11}$ represents the field-aligned current; all are integrated over the north-dawn sector in the simulation for each location in $x$. The units are the same as in Figures 3 and 4.

Figure 5 schematically summarizes the current systems which we found in the simulation and which are necessary to understand the complex field changes. The outermost (red) system 1 represents the perturbed currents of the traditional current wedge, which combines a reduction of the cross-tail current with a diversion into earthward current on the dawnside and tailward current on the duskside (region 1). The system 2 (green) consists of a diversion of radial perpendicular currents into a pair of earthward and tailward currents (feeding both region 1 and 2 currents). Only the dawnside part is shown here. This current system closes here in the ionosphere through north-south currents. A further current system 3 (blue) consists of a dusk-to-dawn current near the equatorial plane, which also reduces the usual cross-tail current, combined with a dawn-to-dusk current at higher latitude. This current system is the simple consequence of the expansion of the dipolarizing region and the associated local reduction of the $B_{x}$ component. This latter closure might be of relevance for possible substorms in Mercury's magnetosphere

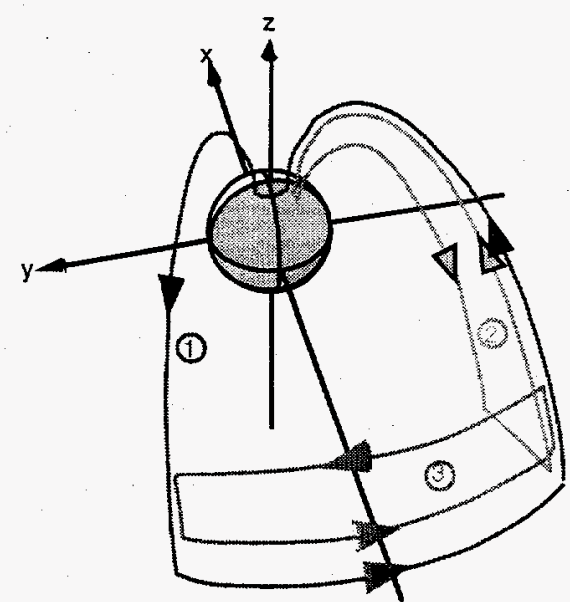

Figure 5. Schematic of the dominant current systems contributing to the diversion of currents in the substorm current wedge. 
where the absence of an appreciable ionosphere inhibits the standard closure of the wedge currents (system 1). Systems 1 and 2 can be associated with the momentum and the forces transferred from the tail to the ionosphere. System 1 corresponds to the earthward transport in the tail leading to an equatorward drag in the ionosphere, while system 2 represents the azimuthal drag away from midnight, corresponding to the azimuthal displacement or bending of field lines in the tail.

\section{Energetic Particle Injections}

The results from the MHD simulation can be used to gain additional information on particle acceleration and energetic particle injections by tracing particles in the MHD fields [Birn et al., 1997b, 1998b]. Fluxes of the energetic particles can be estimated via Liouville's theorem when distribution functions are imposed at the boundaries and for the inital state, consistent with the moments in the MHD simulation and with typical observations in the tail region [e.g., Christon et al., 1991].

Figure 6 shows fluxes obtained this way near the inner edge of the acceleration region at $\mathrm{x}=-10$. Three different locations in $y$ are chosen to demonstrate characteristic variations with local time found in observations: Nearly simultaneous increases in ion and electron fluxes close to midnight, and injection of ions (premidnight) and electrons (postmidnight) that are not accompanied by injections of the other species [Birn et al., 1997a]. Somewhat closer to midnight there are also characteristic injections with delays between the arrivals of the two species (not shown here). These delays as well as the single particle injections can be attributed to a dawn-dusk displacement

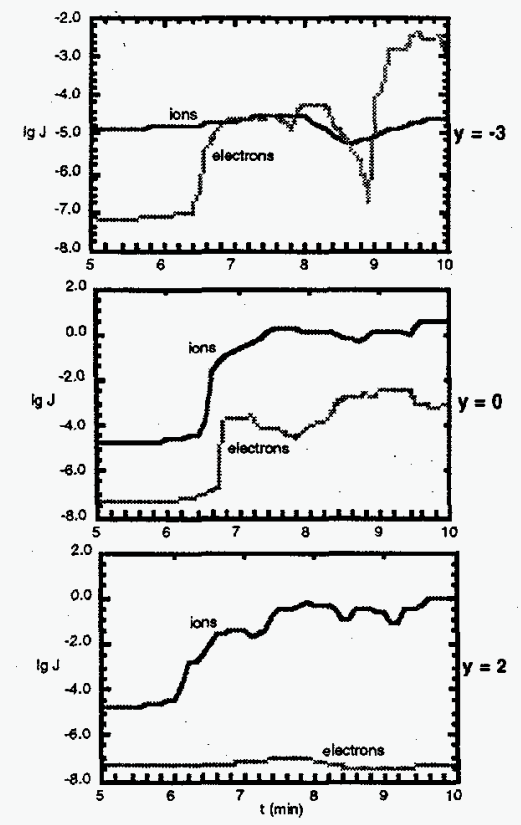

Figure 6. Fluxes of $180 \mathrm{keV}$ protons and electrons, obtained from test particle tracing in the dynamic MHD fields at $x=-10$ and three different locations in $y$ [Birn et al., 1998a]. of the regions of enhanced ion and electron fluxes, resulting from the effects of the cross-tail electric field [Birn et al., 1997a, 1998a]

Figure 7 shows the regions of enhanced fluxes in the equatorial plane at $t=7 \mathrm{~min}$ for electrons and protons of $180 \mathrm{keV}$ with $90^{\circ}$ pitch angle. The figure also includes the location of the neutral line (white line) and contours of the cross-tail electric field strength, to indicate where the acceleration takes place. The ion and electron injection regions show a common central part, which is located at the earthward end of the region of strong $E_{y}$. Closest to the Earth, the enhanced-flux regions separate, ions toward dusk, electrons toward dawn, as expected from their drifts. For the electrons, as well as for the ions, we find that the regions of the strongest flux enhancement are well earthward of the neutral line (located near $x=-23 R_{E}$ ). Both injection regions also have tailward edges well earthward of the neutral line.

\section{Summary and Discussion}

We have presented major results of recent investigations of substorm tail dynamics, based on resistive MHD simulations [Birn and Hesse, 1996, 1998] and test particle orbits in the electric and magnetic fields of this simulation [Birn et al., 1997b, 1998a]. The simulation includes the

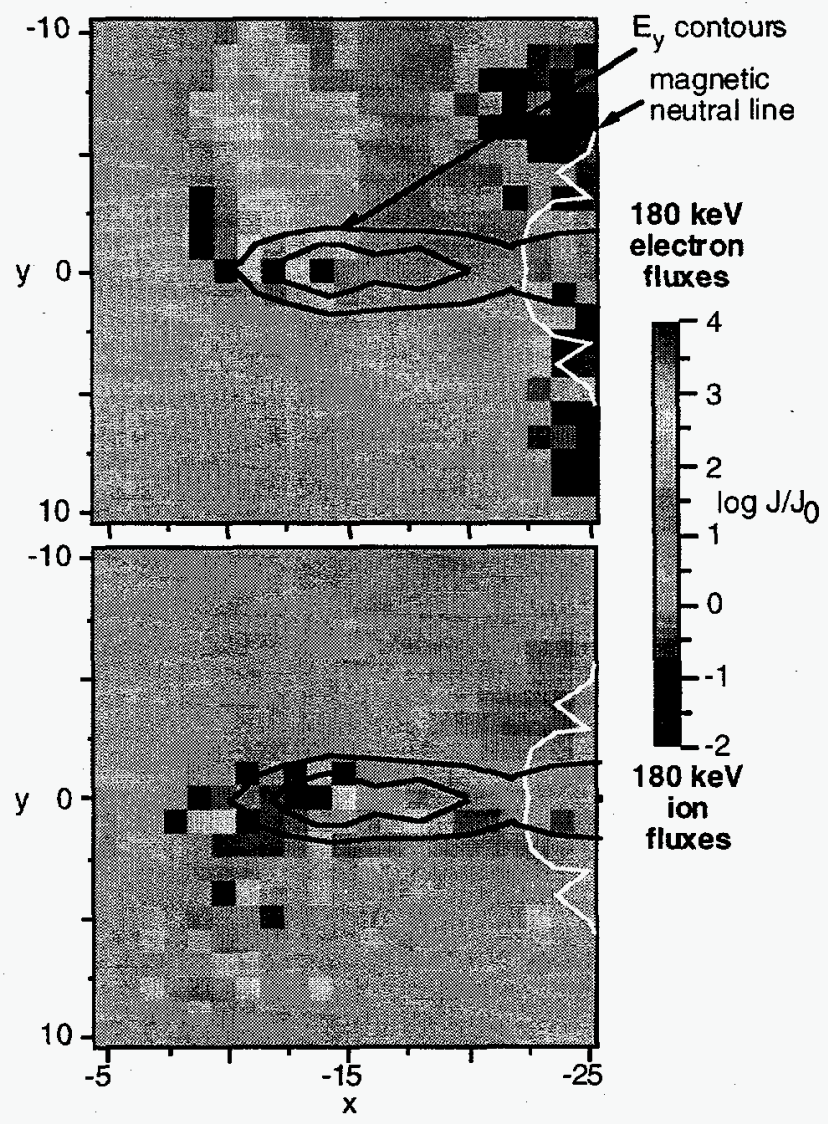

Figure 7. Fluxes of $180 \mathrm{keV}$ protons and electrons in the $x$, $y$ plane, obtained from test particle tracing in the dynamic MHD fields, at $t=7 \mathrm{~min}$ (after Birn et al. [1998a]). 
formation of a thin current sheet in the transition region between a dipole field and the tail as the consequence of an externally applied electric field during a simulated growth phase. The dynamic state self-consistently combines the two main features commonly considered for particle acceleration in the inner magnetotail, a near-Earth $\mathrm{x}$-type magnetic neutral line, located approximately at $18 R_{\mathrm{E}}$ from the near-Earth boundary of the simulation box $\left(23 R_{\mathrm{E}}\right.$ from the center of the dipole), and the collapse and dipolarization of the innermost tail region earthward of the neutral line. This inner region is not only the region of the strongest electric field and dipolarization (usually interpreted as current "disruption") but also the site of flow braking and diversion, causing the field distortions and the current diversion associated with the substorm current wedge.

The simulation results demonstrate that the complex magnetic field changes cannot be understood by only one perturbed current system, the traditional current wedge (system 1 in Figure 5), alone. One additional system is associated with a diversion of radial tail currents into fieldaligned currents. It combines both region 1 and region 2 field-aligned currents (system 2 in Figure 5) and may close through equatorward currents in the ionosphere. It corresponds to a transfer of azimuthal momentum from the magnetotail to the ionosphere, providing an azimuthal drag, whereas system 1 transmits earthward momentum leading to an equatorward drag on the ionosphere.

Two additional systems are confined to the tail. System 3 (Figure 5) is the direct consequence of the expansion of the current sheet in the dipolarizing region. It combines a reduction of the cross-tail current near the equatorial plane with an increase of the duskward current at higher latitude, contributing to the field-aligned currents only locally. A further current loop (not shown in Figure 5) consists of a tailward/earthward pair of field-aligned currents that close in the tail through perpendicular currents that are radial near the equatorial plane but roughly north-south oriented at higher latitudes.

The simulation results strongly suggest the following substorm scenario illustrated by Figure 8 . Substorm onset in the tail is closely associated with the occurrence of reconnection in the tail region between $\sim 20$ and $30 R_{E}$, perhaps after a microinstability is triggered or some other dissipation threshold is exceeded by the formation of a thin current sheet. Reconnection not only leads to plasmoid formation and tailward ejection but also to the generation of an earthward flow burst. The slow-down of this flow in the transition region to the dipole leads to a compression of the magnetic field and hence dipolarization, which may propagate tailward due to a pile-up effect. The diversion of the flow around the Earth and the shear at the outer edges of the flow lead to an increased shear of the magnetic field, that is, the build-up of field-aligned currents:-The magnetic shear generated on the outside of the flow region corresponds to earthward flowing field-aligned currents on the dawnside and tailward flowing currents on the duskside, that is, the region 1 sense of the substorm wedge currents. Here "outside" refers to farther away from midnight in the region of earthward flow, radially outward in the region where the flow is diverted into the azimuthal direction, and farther away from the equatorial plane in either region. It is interesting to note that the flow shear

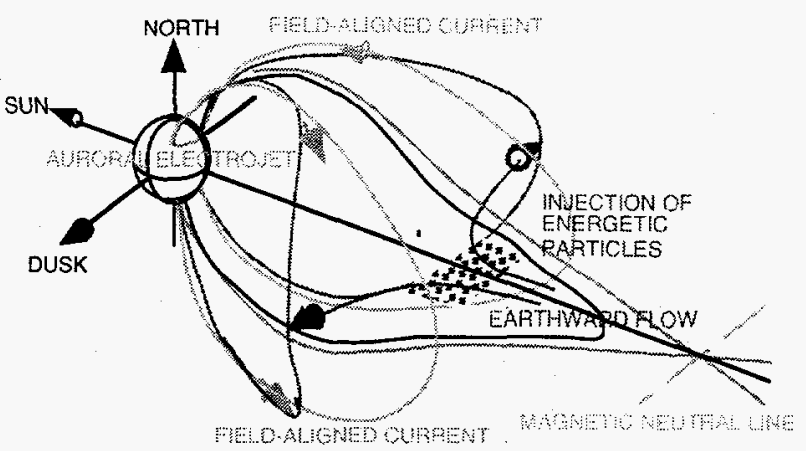

Figure 8. Schematic of the association between an earthward flow burst and the field distortions leading to the fieldaligned currents of the substorm current wedge. The slowdown of this flow provides magnetic field compression and dipolarization. The shear at the outer edges of the flow causes magnetic shear, that is, field-aligned currents. The strong electric field associated with the earthward flow and the dipolarization is also the source of particle energization and injections.

on the inner (i. e., earthward) side of the region where the flow is diverted around the Earth generates an opposite magnetic shear corresponding to region 2 type fieldaligned currents, so that the same flow field might be responsible for an increase of both region 1 and region 2 currents.

The direct effects of the braking via inertia currents last only as long as the flow burst, that is, a few minutes. The effects of the field distortion and the associated pressure gradient changes, however, last longer, until the field-aligned currents corresponding to the twisted or sheared magnetic field get dissipated in the ionosphere or in the auroral acceleration region.

The earthward flow burst and the collapse of the field toward a more dipolar field at its earthward edge is associated with a strong electric field, which is also responsible for the acceleration of energetic particles and their injection into the inner magnetosphere [Birn et al., 1997b, 1998a]. The fluxes obtained from test particle orbits in the MHD fields show excellent agreement with observations of substorm injections, in the magnitude of the typical flux increases as well as in their characteristic energy dependence. This indicates that smaller-scale fluctuations, which are absent in the MHD simulation, have only minor effects on the ion fluxes and that the mechanisms of neutral line acceleration and field collapse or dipolarization of the inner tail (which is similar to the "convection surge mechanism" [Quinn and Southwood, 1982; Mauk, 1986]) are the major mechanisms responsible for particle acceleration and injection in the inner magnetosphere and tail. In the present model these mechanisms are closely coupled through the underlying large-scale tail instability, triggered by the local breakdown of ideal MHD.

Acknowledgments. This work was carried out under the auspices of the United States Department of Energy with support from its Office of Basic Energy Sciences and from NASA. 


\section{References}

Baumjohann, W., G. Paschmann, T. Nagai, and H. Lühr, Superposed epoch analysis of the substorm plasma sheet, $J$. Geophys. Res., 96, 11,605, 1991.

Birn, J., Magnetotail equilibrium theory: The general threedimensional solution, J. Geophys. Res., 92, 11,101, 1987.

Birn, J., and M. Hesse, The substorm current wedge and fieldaligned currents in MHD simulations of magnetotail reconnection, J. Geophys. Res., 96, 1611, 1991.

Birn, J., and M. Hesse, Details of current disruption and diversion in simulations of magnetotail dynamics, $J$. Geophys. Res., in press, 1996.

Birn, J., M. F. Thomsen, J. E. Borovsky, G. D. Reeves, D. J. McComas, and R. D. Belian, Characteristic plasma properties of dispersionless substorm injections at geosynchronous orbit, $J$. Geophys. Res., 102, 2309, 1997a.

Birn, J., M. F. Thomsen, J. E. Borovsky, G. D. Reeves, D. J. McComas, R. D. Belian, and M. Hesse, Substorm ion injections: Geosynchronous observations and test particle orbits in three-dimensional dynamic MHD fields, J. Geophys. Res., 102, 2325, $1997 \mathrm{~b}$.

Birn, J., M. F. Thomsen, J. E. Borovsky, G. D. Reeves, D. J. McComas, R. D. Belian, and M. Hesse, Substorm electron injections: Geosynchronous observations and test particle simulations, J. Geophys. Res., 103, 9235, 1998a.

Birn, J., M. Hesse, G. Haerendel, W. Baumjohann, and K. Shiokawa, Flow braking and the substorm current wedge, $J$. Geophys. Res., submitted, 1998b.

Christon, S. P., D. J. Williams, and D. G. Mitchell, Spectral characteristics of plasma sheet ion and electron populations during disturbed geomagnetic conditions, J. Geophys. Res., 96, 1, 1991.

Haerendel, G., Disruption, ballooning or auroral avalanche On the cause of substorms, in Substorms 1, Proc. of the First Int. Conf. on Substorms (ICS-l), Kiruna, Sweden, ESA Publ., SP-335, 417, 1992.

Hesse, M., and J. Birn, On dipolarization and its relation to the substorm current wedge, J. Geophys. Res., 96, 19,417, 1991.

Hones, E. W., Jr., Substorm processes in the magnetotail: Comments on "On hot tenuous plasma fireballs and boundary layers in the Earth's magnetotail" by L. A. Frank, L. L. Ackerson, and R. P. Lepping, J. Geophys. Res., 82, 5633, 1977.

Huang, C. Y., Frank, L. A., Rostoker, G. Fennell, J., and Mitchell, D. G., Nonadiabatic heating of the central plasma sheet at substorm onset, J. Geophys. Res., 97, $1481,1982$.

Lui, A. T. Y., A. Mankofsky, C.-L. Chang, K. Papadopoulos, and C. S. Wu, A current disruption mechnism in the neutral sheet: A possible trigger for substorm expansions, Geophys. Res. Lett., 17, 745, 1990.

Mauk, B. H., Quantitative modeling of the "convection surge" mechanism of ion acceleration, J. Geophys. Res., $91,13,423,1986$.

McPherron, R. L., C. T. Russell, and M. A. Aubry, Satellite studies of magnetospheric substorms on August 15, 1968, 9. Phenomenological model for substorms, J. Geophys. Res., 78, 3131, 1973.

McPherron, R. L., A. Nishida, and C. T. Russell, Is near-Earth current sheet thinning the cause of auroral substorm onset?, in Quantitative Modeling of Magnetosphere-
Ionosphere Coupling Processes, edited by Y. Kamide and R. A. Wolf, p. 252, Kyoto Sangyo University, Kyoto, Japan, 1987.

Quinn, J. M., and D. J. Southwood, Observations of parallel ion energization in the equatorial region, J. Geophys. Res., 87, 10,536, 1982.

Reeves, G. D., New perspectives on substorm injections, this issue, 1998

Sanny, J., R. L. McPherron, C. T. Russell, D. N. Baker, T. I. Pulkkinen, and A. Nishida, Growth-phase thinning of the near-Earth current sheet during the CDAW 6 substorm, $J$. Geophys. Res., 99, 5805, 1994.

Schindler, K., and J. Bim, On the cause of thin current sheets in the near-Earth magnetotail and their possible significance for magnetospheric substorms, J. Geophys. Res., $98,15,477,1993$.

Scholer, M., and A. Otto, Magnetotail reconnection: Current diversion and field-aligned currents, Geophys. Res. Lett, $18,733,1991$.

Shiokawa, K., W. Baumjohann, and G. Haerendel, Braking of high-speed flows in the near-Earth tail, Geophys. Res. Lett., 24, 1179, 1997.

Shiokawa, K., G. Haerendel, and W. Baumjohann, Azimuthal pressure gradient as driving force of substorm currents, Geophys. Res. Lett., 25, 959, 1998. 
CROSS-TAIL ELECTRIC FIELD $E_{y}, t=8 \mathrm{~min}$

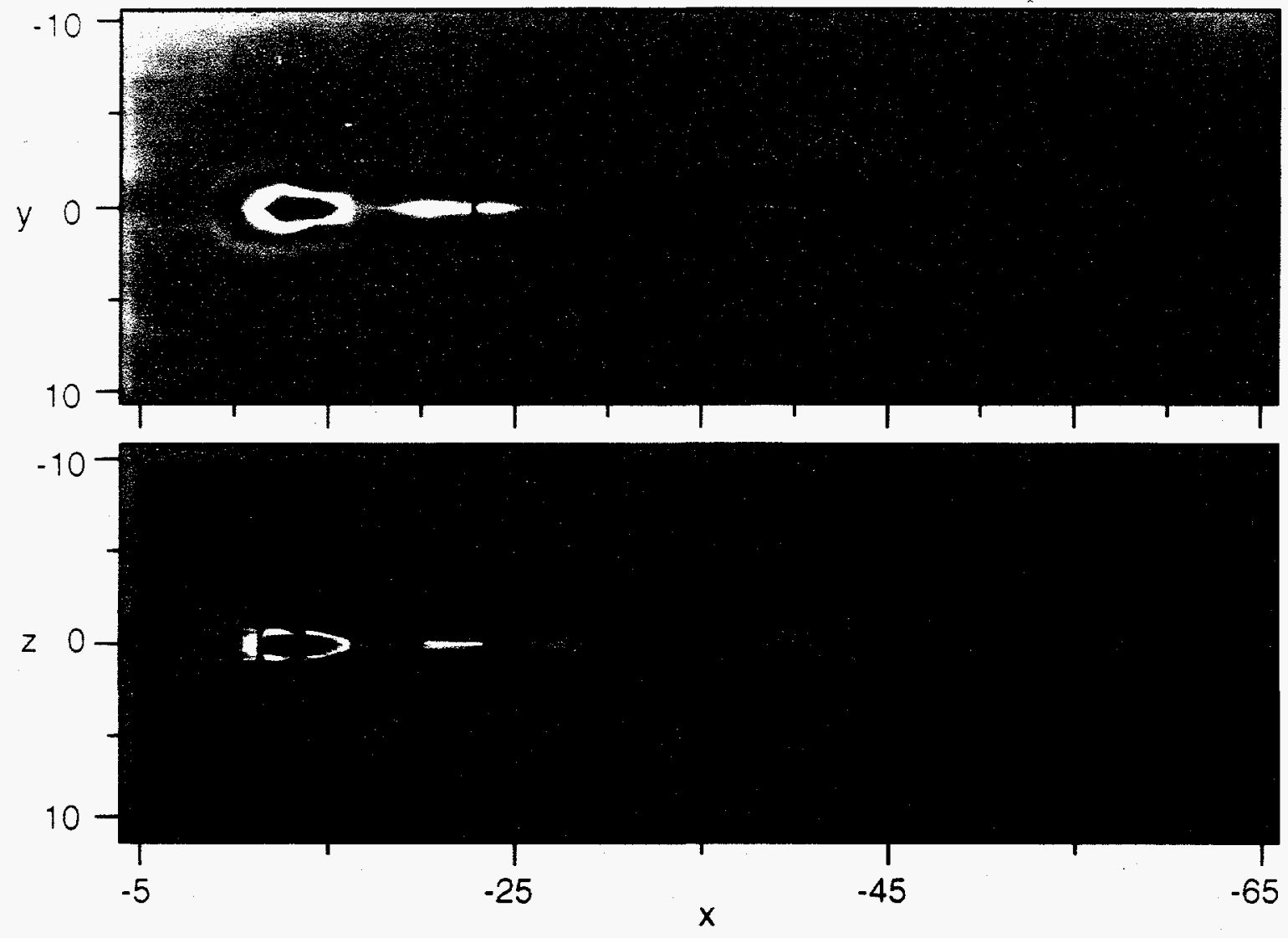

$E_{y}(m \vee / m)$

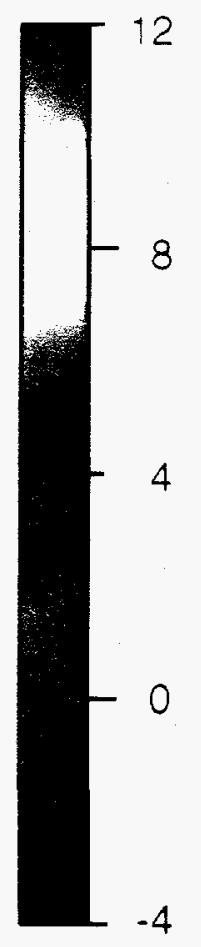

TEMPERATURE, VELOCITY VECTORS, \& NEUTRAL LINE $t=8 \mathrm{~min}$
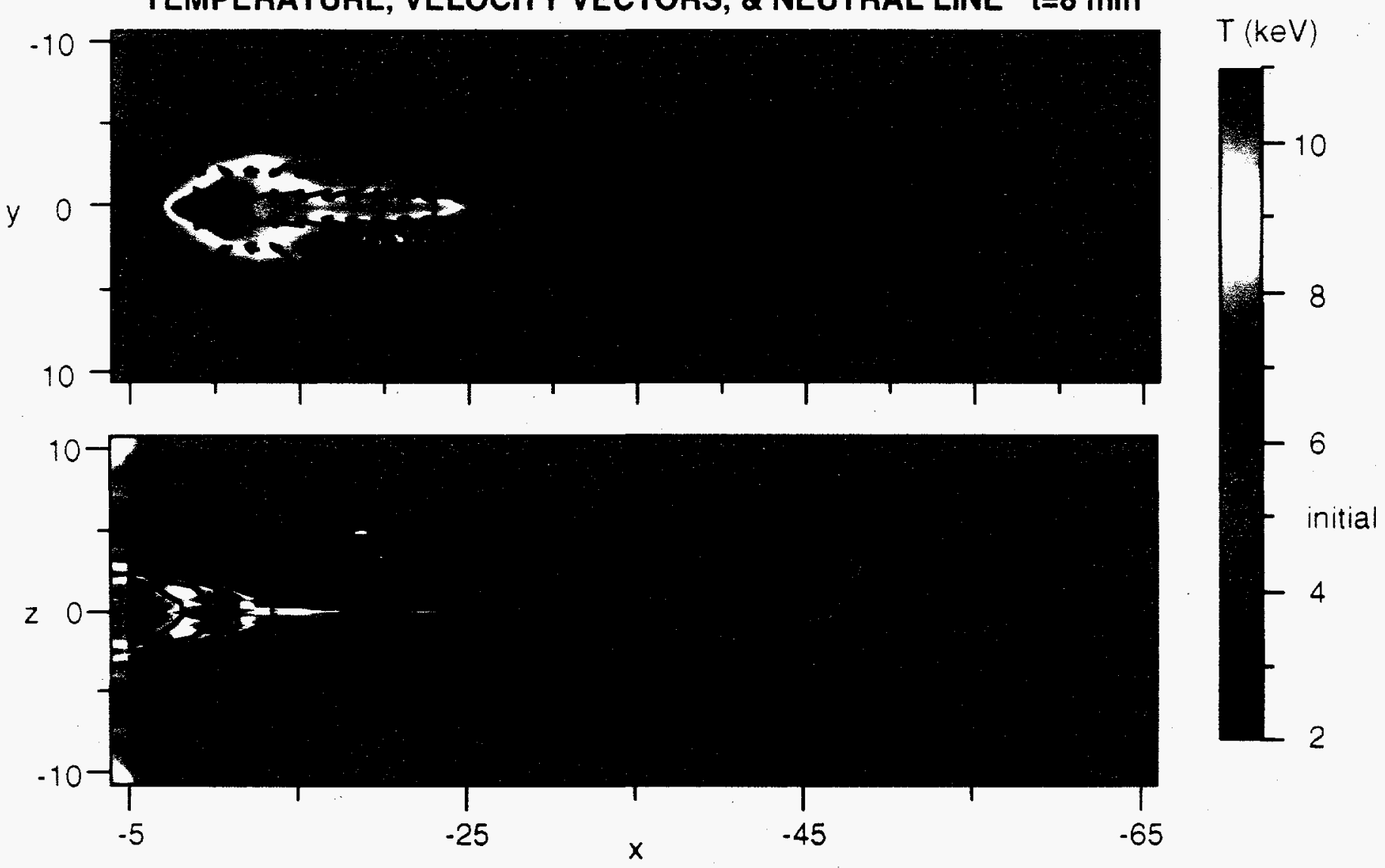

Ris. 1 


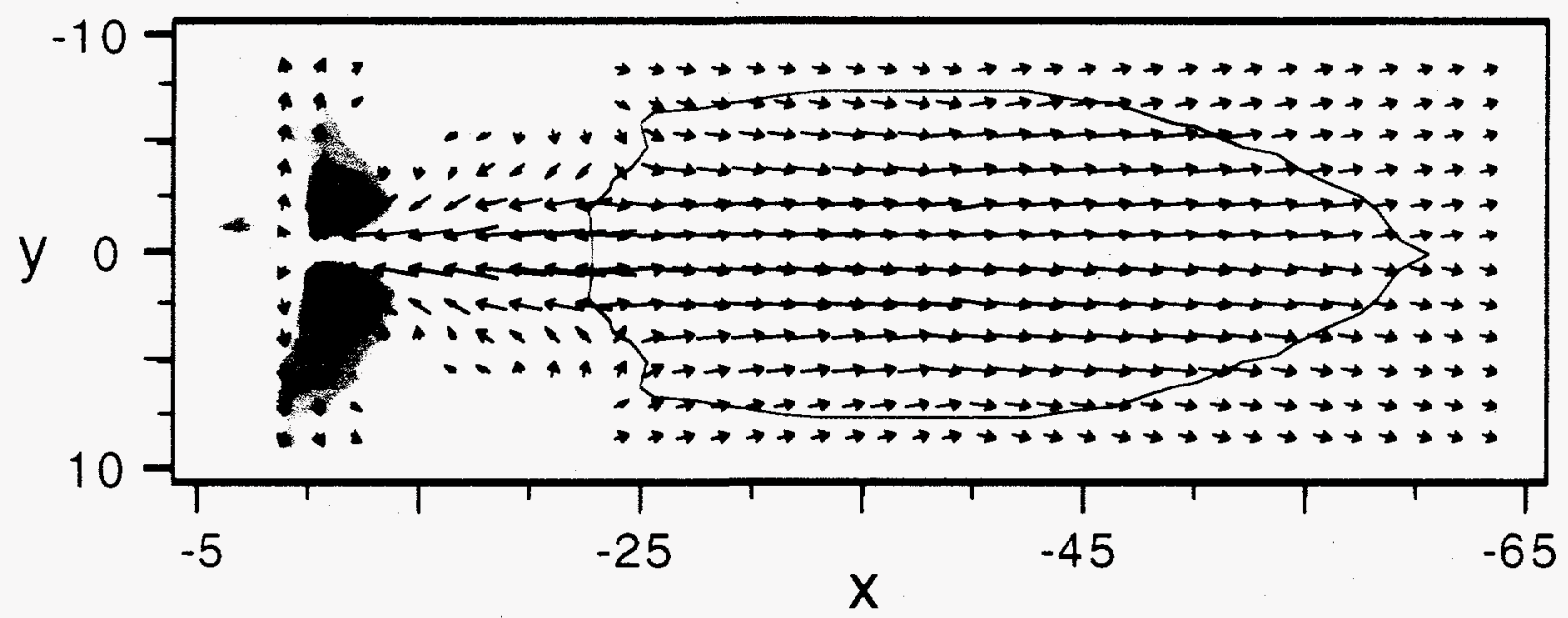

Rig. 2 

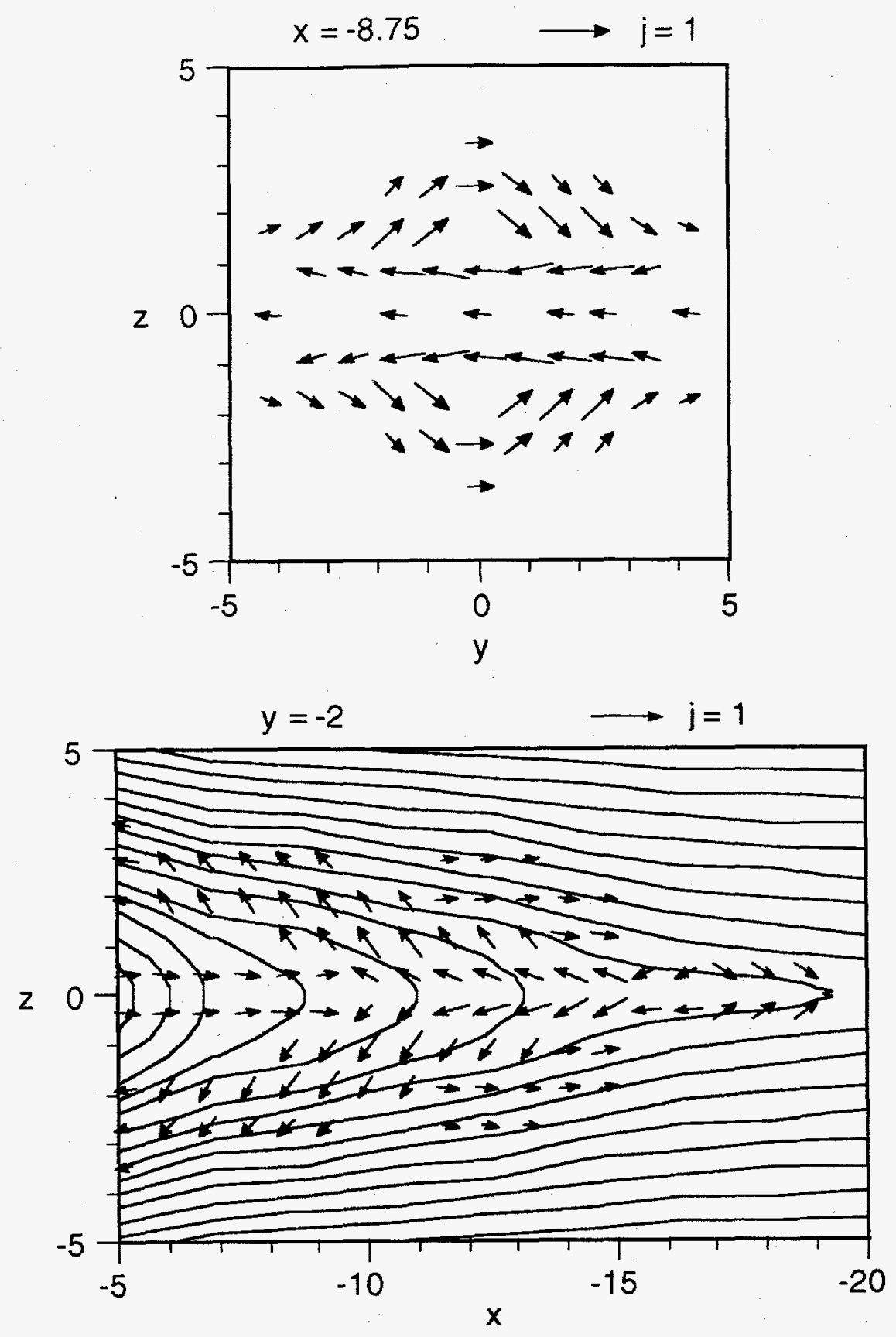


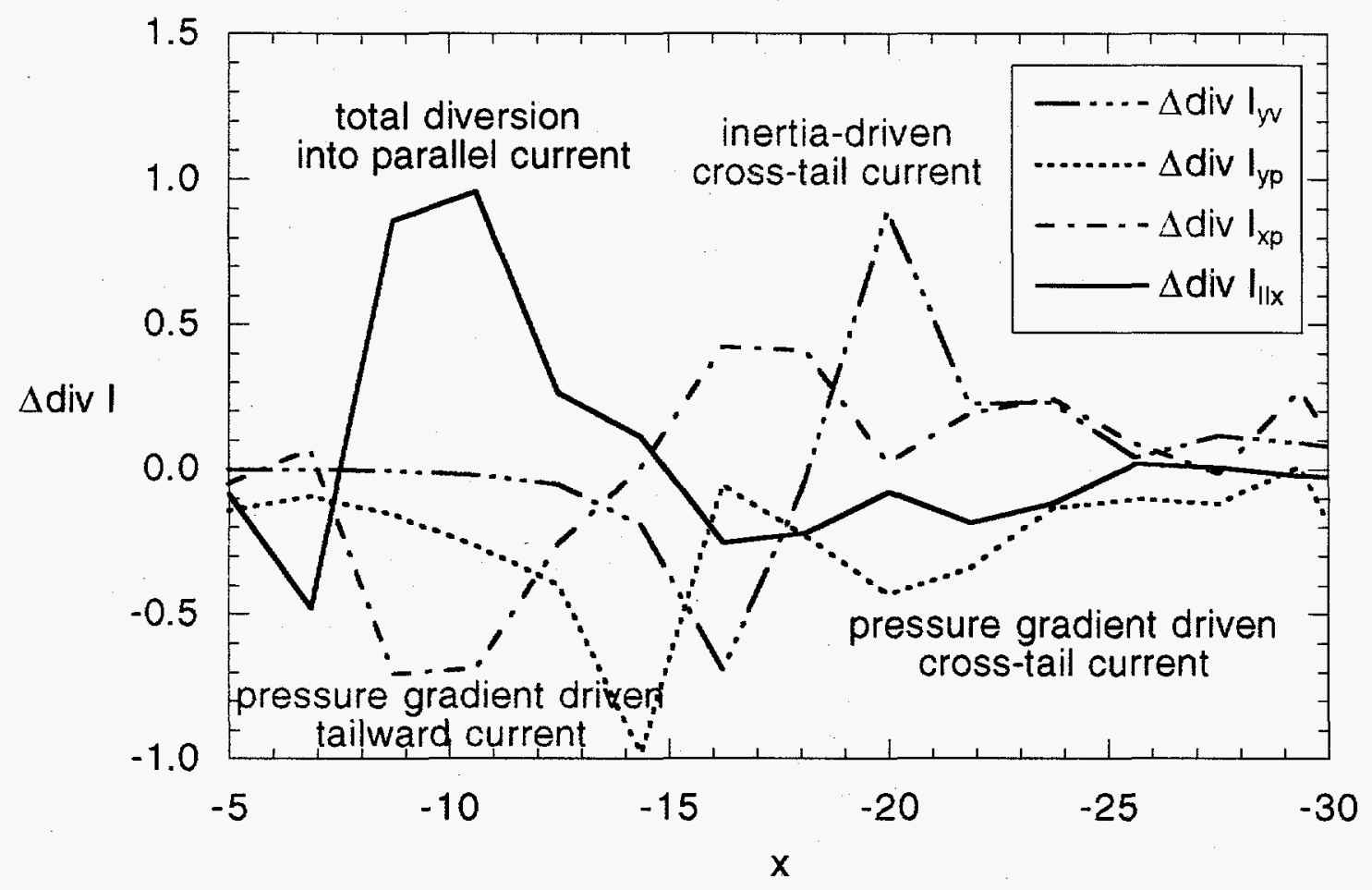




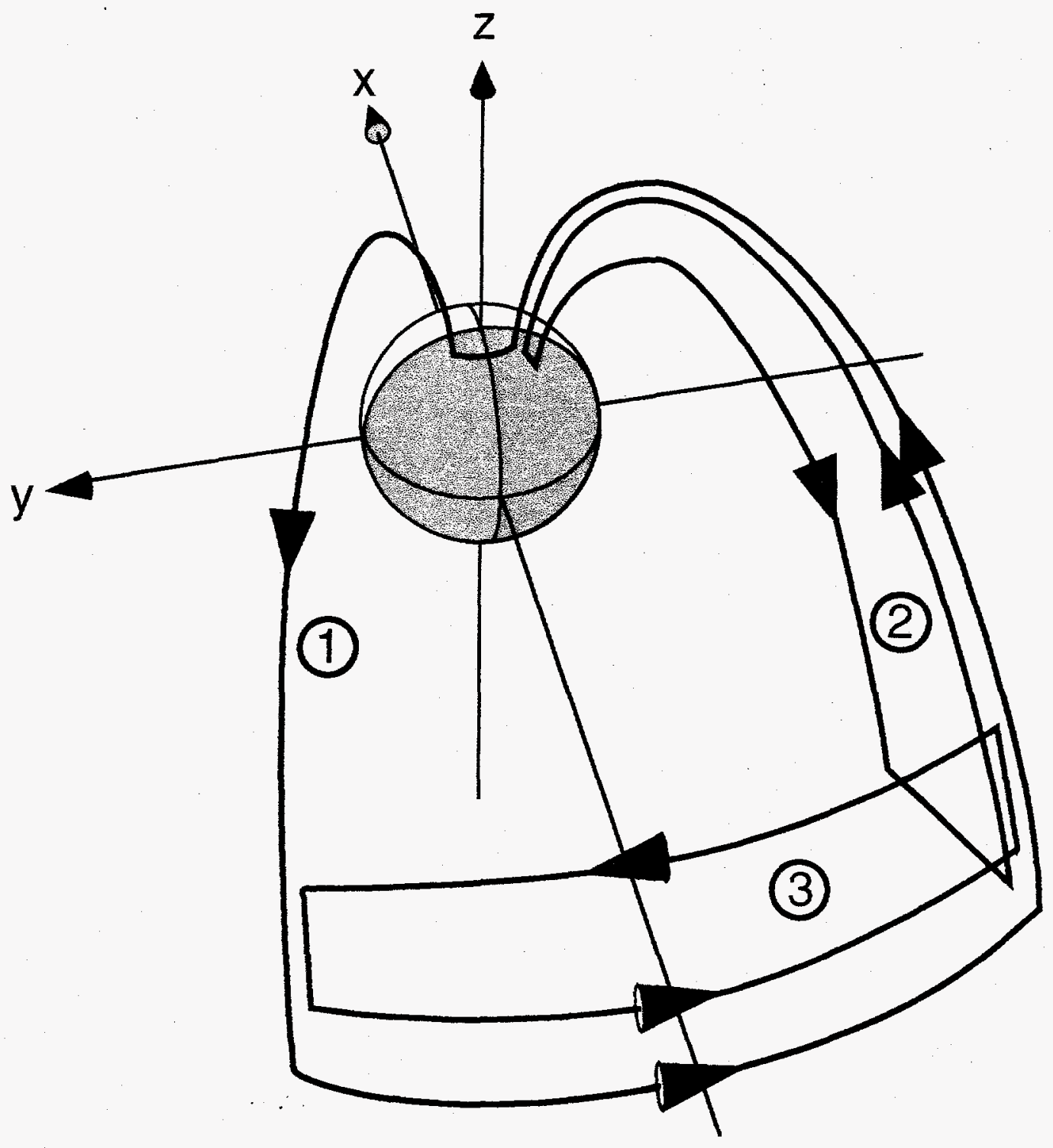



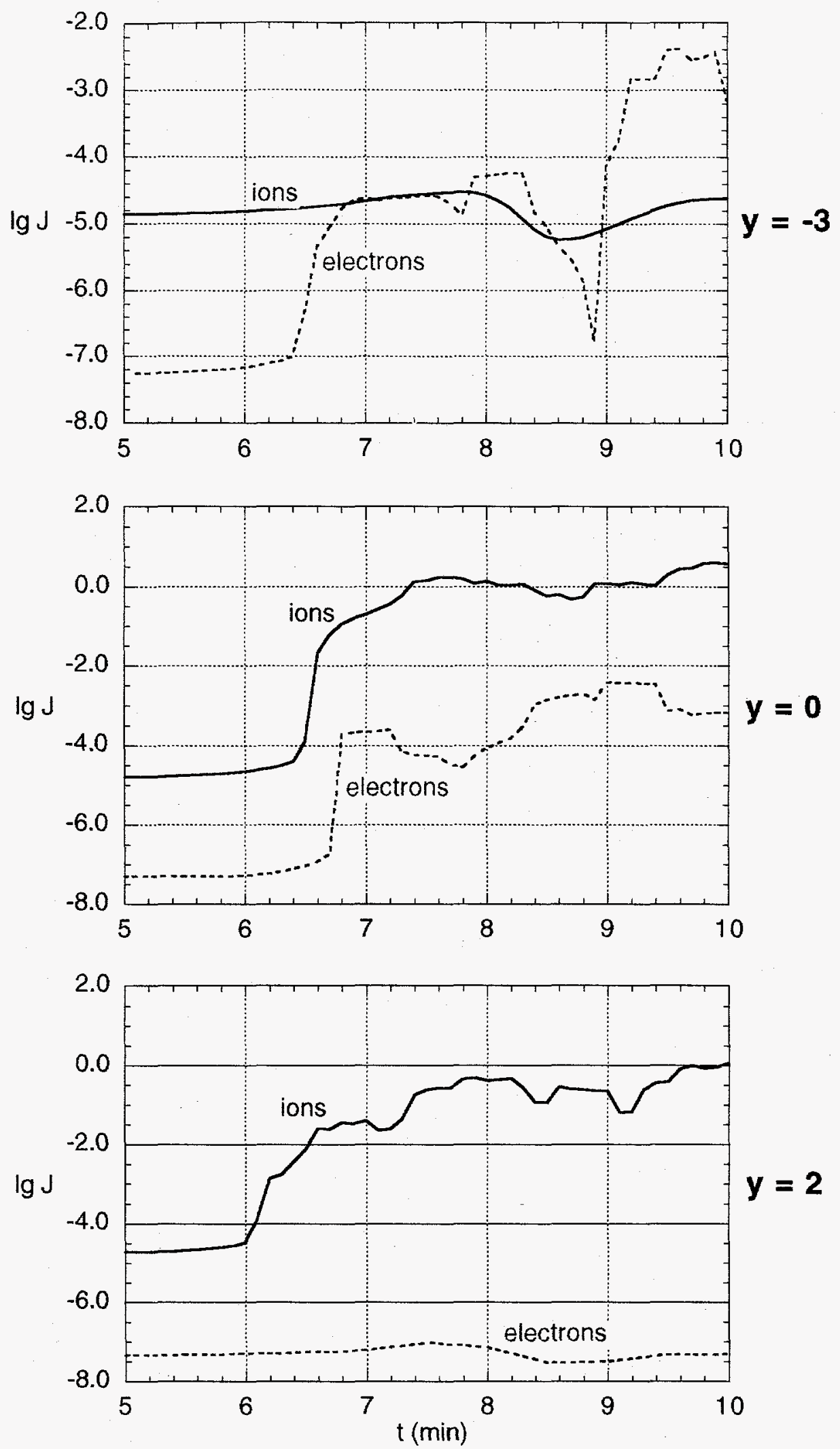


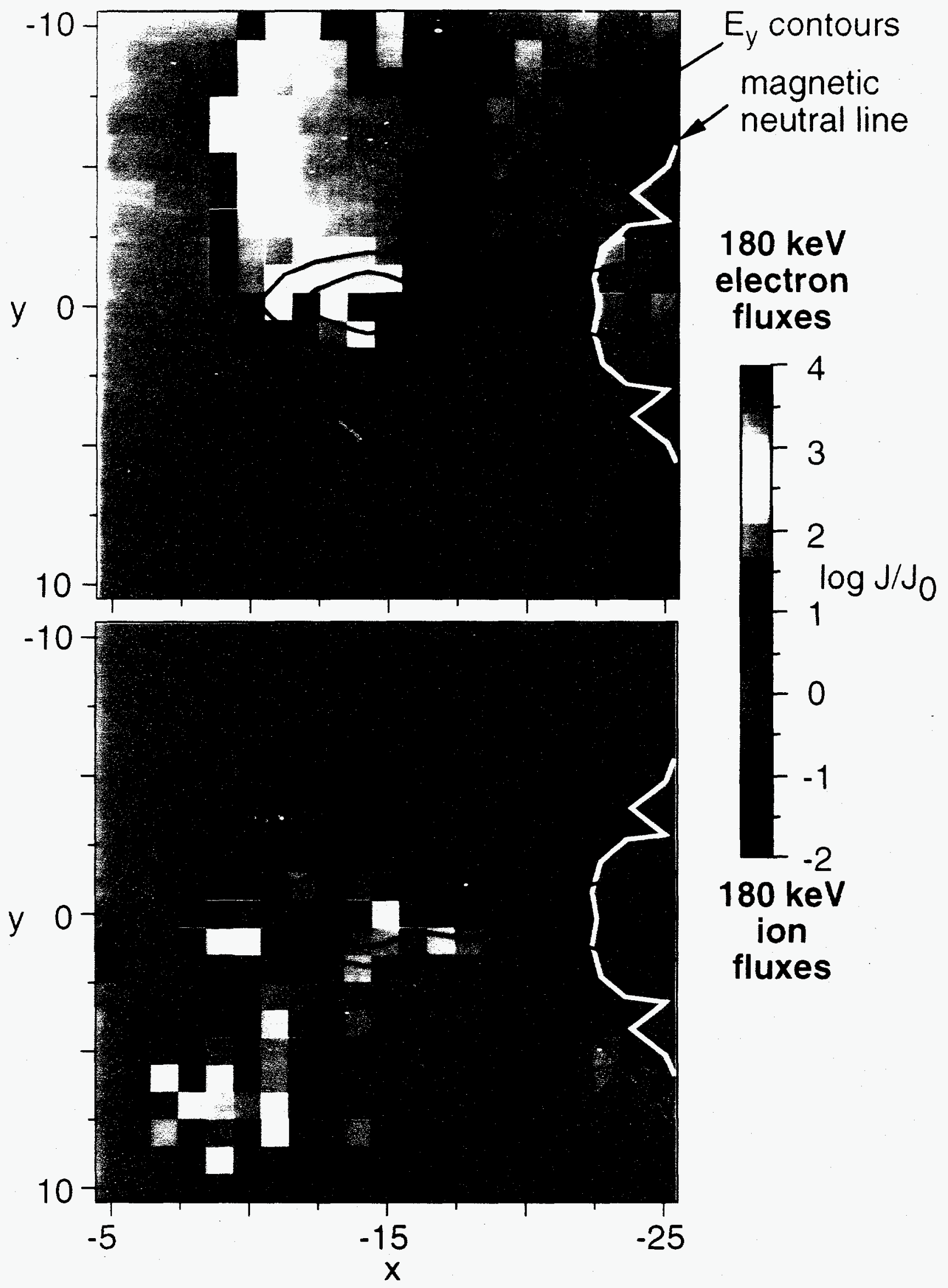

Fig. 7 


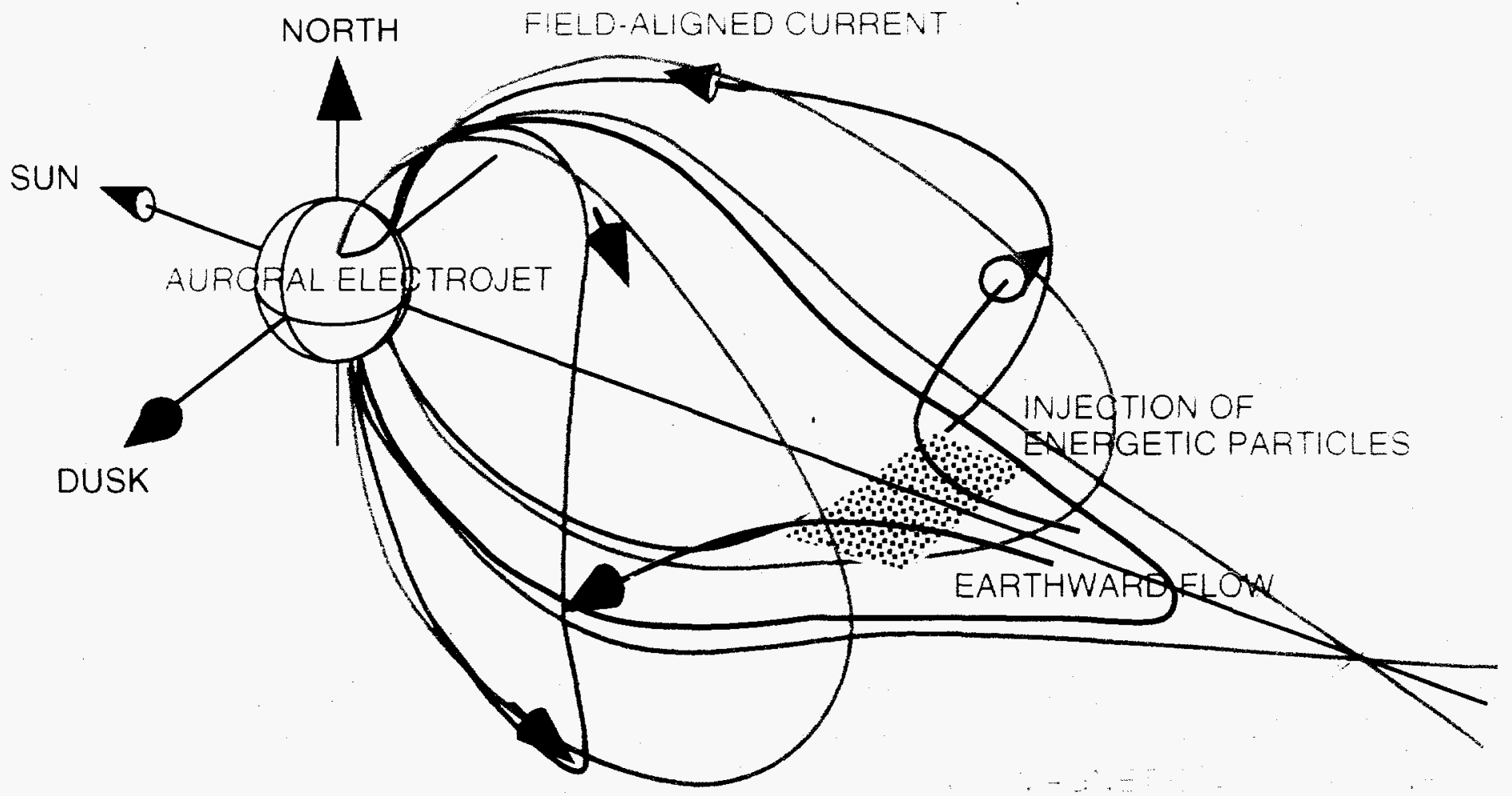

FIELD-ALIGNED CURRENT

$F_{j} .8$ 